\title{
Intravesical Mitomycin-C-Induced Interstitial Pneumonia
}

\author{
Hiroshi Wada a, b Yasutaka Nakano ${ }^{a}$ Hideto Yamada ${ }^{a}$ Tatsuyoshi Saiga ${ }^{c}$ \\ Akira Yamanaka $^{d}$ Naoki Sakai ${ }^{b}$ \\ a Division of Respiratory Medicine, Department of Internal Medicine, Shiga University of Medical Science, and \\ Departments of ${ }^{b}$ Respiratory Medicine, ${ }^{c}$ Pathology and ${ }^{d}$ Chest Surgery, Otsu Red Cross Hospital, Otsu, Japan
}

\section{Established Facts}

- Systemic mitomycin C administration may lead to severe complications, including interstitial pneumonia.

\section{Novel Insights}

- Intravesical mitomycin C administration may lead to fatal interstitial pneumonia, although it has been considered a safe treatment.

\section{Key Words}

Drug-induced lung toxicity $\cdot$ Interstitial lung disease • Intravesical drug administration - Mitomycin C

\begin{abstract}
An 84-year-old man with no evidence of pre-existing interstitial pneumonia developed fatal interstitial pneumonia and respiratory failure that could only be explained as an adverse effect of intravesical mitomycin C chemotherapy. He had undergone transurethral resection of bladder cancer 3 times, followed by intravesical mitomycin C chemotherapy 1 month later. He had received intravesical mitomycin $C$ chemotherapy every week for 2 months, and he had complained of dyspnea on exertion 5 days before the last intravesical mitomycin C chemotherapy session. Interstitial infiltration was detected in both the upper and lower lobes of the left lung on the day of the last instillation of mitomycin C. Despite dis-
\end{abstract}

continuation of mitomycin $\mathrm{C}$ and administration of methylprednisolone, his condition deteriorated and he died. Diagnostic evaluation of other causes of pneumonia, including infection and collagen-vascular diseases, was negative. The autopsy demonstrated diffuse alveolar damage in the lung; there were no remarkable findings in other organs. To our knowledge, this is the first pathologically confirmed case of fatal interstitial pneumonia due to intravesical mitomycin $C$ chemotherapy.

Copyright $\odot 2010$ S. Karger AG, Basel

\section{Introduction}

Mitomycin C, an antineoplastic antibiotic derived from Streptomyces caespitosis, is widely used in the treatment of many malignant tumors. Instillation of mitomycin $\mathrm{C}$ into the bladder (intravesical mitomycin $\mathrm{C}$ chemo-

\section{KARGER \\ Fax +4161306 1234 E-Mail karger@karger.ch} www.karger.com (c) 2010 S. Karger AG, Basel 0025-7931/10/0803-0256\$26.00/0

Accessible online at:

www.karger.com/res
Hiroshi Wada, MD

Division of Respiratory Medicine, Department of Internal Medicine

Shiga University of Medical Science

Seta Tsukinowa-cho, Otsu, Shiga, 520-2192 (Japan)

Tel. +81 77548 2213, Fax +81 77543 5839, E-Mail hwada@ belle.shiga-med.ac.jp 
therapy) is a common procedure following transurethral tumor resection (TUR), with the aim of preventing recurrence of bladder cancer [1]. Intravesical mitomycin C chemotherapy is recognized as a safer treatment than systemic chemotherapy since the urothelium serves as a barrier that limits the absorption of drugs into systemic circulation [2]. Here, we report a fatal case of interstitial pneumonia (diffuse alveolar damage) following intravesical mitomycin C chemotherapy after TUR.

\section{Case Report}

An 84-year-old Japanese man who had previously undergone TUR twice for superficial bladder cancer underwent the third TUR procedure in August 2006. He also underwent instillation of mitomycin C (20 mg) into the bladder (intravesical mitomycin $\mathrm{C}$ chemotherapy) in September. Thereafter, he continued to receive intravesical mitomycin $\mathrm{C}$ chemotherapy every week. Although he complained of dyspnea on exertion starting on November 2 , he had completed 8 sessions in total on November 7. After the last instillation, he underwent chest X-ray and CT, which showed ground glass opacity, and he was referred to our hospital on November 7.

He had no history of smoking or drinking. He had not been exposed to any toxic dusts, fumes, asbestos, birds or other animals. He had a history of right pleuritis of unknown cause 11 years earlier and a gastric ulcer 1 year earlier. Chest CT obtained prior to intravesical mitomycin $\mathrm{C}$ chemotherapy did not show any evidence of interstitial pneumonia.

On admission, his body temperature was $37.5^{\circ} \mathrm{C}$, blood pressure was $149 / 71 \mathrm{~mm} \mathrm{Hg}$, pulse rate was 75 beats/min and respiratory rate was 20 times/min. Superficial lymph nodes were not palpable. Findings on chest examination were normal without inspiratory crackles over the bilateral lung fields. Arterial blood gas analysis at rest (with $2 \mathrm{l} / \mathrm{min}$ of oxygen) showed $\mathrm{PaO}_{2} 87.8$ $\mathrm{mm} \mathrm{Hg}$ and $\mathrm{PaCO}_{2} 39.2 \mathrm{~mm} \mathrm{Hg}$. Laboratory data on admission demonstrated slight anemia ( $\mathrm{Hb} 10.5 \mathrm{~g} / \mathrm{dl})$, leukocytosis $(14,200 /$ $\mathrm{cm}^{3}$ ) without eosinophilia and marked elevation of CRP (16.7 $\mathrm{mg} / \mathrm{dl}$ ). Urinary antigen for Legionella was negative. Serum KL6 (Krebs von den Lungen-6) and SP-D (surfactant protein D) concentrations were within normal ranges. Only a slightly elevated titer of anti-scleroderma antibody (anti Scl-70 antibody) was found on screening for autoantibodies. Chest X-ray (fig. 1a) showed ground glass opacity in the left lower field. Chest CT (fig. 1b) discovered ground glass opacity with a crazy-paving appearance and consolidations in the left upper and left lower lobes. There was no change in loculated pleural effusion and pleural thickening in the right lung, which had been found during the previous examination before the intravesical mitomycin C chemotherapy.

Bronchoalveolar lavage was performed from left anterior basal bronchus (left B8) on the day of admission. Saline $(150 \mathrm{ml})$ was instilled into the left lower lobe bronchus in the right lateral decubitus position, and only $11 \%$ of the volume was recovered. Bronchoalveolar lavage fluid appeared bloody with a total cell count of $3.0 \times 10^{5} / \mu$ l (neutrophils $81 \%$, lymphocytes $6 \%$, eosinophils $4 \%$, macrophages 9\%) and showed an elevated number of neutrophils. Neither bacteria nor atypical cells were found in the bronchoalveolar lavage fluid. Transbronchial lung biopsy was performed in the left lower lung, and indicated diffuse alveolar damage (DAD), including the hyaline membrane. A lymphocyte stimulation test, performed against mitomycin $\mathrm{C}$ in his blood, demonstrated nonsignificant proliferation of lymphocytes with a stimulation index of 0.81 .

Considering the possibility of infectious disease, we started antibiotics (pazufloxacin) and discontinued mitomycin C. On the second day, the patient's respiratory condition deteriorated and ground glass opacity appeared to have spread throughout the left lung on chest X-ray. Therefore, we added a 3-day course of intravenous methylprednisolone (steroid pulse therapy: 1,000 mg/ day). On the third day, a neutrophil elastase inhibitor, sivelestat sodium, was also added. Although the criteria of acute respiratory distress syndrome were not met at the first visit, it was suspected that these criteria were met during the course of hospitalization. Despite the administration of these drugs, the patient deteriorated progressively and died of acute respiratory distress syndrome on the 6th day.

Upon autopsy, irregular thickening of the urinary bladder mucosa was found. Histologically, transitional epithelium covered the superficial layer. The bladder had a few ulcers and atypical cells were found, indicating carcinoma in situ. The lungs were markedly enlarged: the right lung was $650 \mathrm{~g}$ and the left lung was $1,110 \mathrm{~g}$. Macroscopic findings showed strong pleural adhesions in the right lung and hemorrhage in the left lung. Microscopic findings of the lungs showed hyaline membrane with marked fibrin deposition, desquamated alveolar epithelium, bleeding into the alveolar space, infiltration of inflammatory cells to the alveolar walls, and thickening of the alveolar walls (fig. 1c). Neither fungus nor intranuclear inclusion bodies were found. There was no evidence of infection and the findings were diagnosed as DAD. There were no remarkable findings other than those in the lungs. The cause of death was identified as respiratory failure due to DAD.

\section{Discussion}

The present case demonstrated that intravesical mitomycin C chemotherapy may be associated with fatal interstitial pneumonia. Therefore, intravesical mitomycin $\mathrm{C}$ chemotherapy must be considered as a treatment that can cause or contribute to fatal interstitial pneumonia. This is important information since intravesical mitomycin C chemotherapy is associated with a lower incidence of systemic adverse effects and considered a safer treatment than systemic chemotherapy [3].

The diagnosis of intravesical mitomycin $\mathrm{C}$ chemotherapy-induced interstitial pneumonia was based on the fact that this patient had no history of pre-existing interstitial pneumonia and did not receive any drug other than mitomycin $\mathrm{C}$ before the development of interstitial pneumonia. Infectious diseases and collagen vascu- 
Fig. 1. Chest X-ray and CT on admission and autopsy findings of the left lung. a Chest X-ray showed ground glass opacity in the left lower lung field and pleural thickening in the right lung. b Chest CT showed ground glass opacity with a crazypaving appearance in the left upper lobe and left lower lobe. Pleural thickening and old inflammatory change were also seen in the right lung. c Autopsy findings included hyaline membrane with deposition of marked fibrin, desquamated alveolar epithelium, bleeding in the alveolar space, infiltration of inflammatory cells into the alveolar walls, and thickening of the alveolar walls (hematoxylin and eosin stain $\times 100)$.
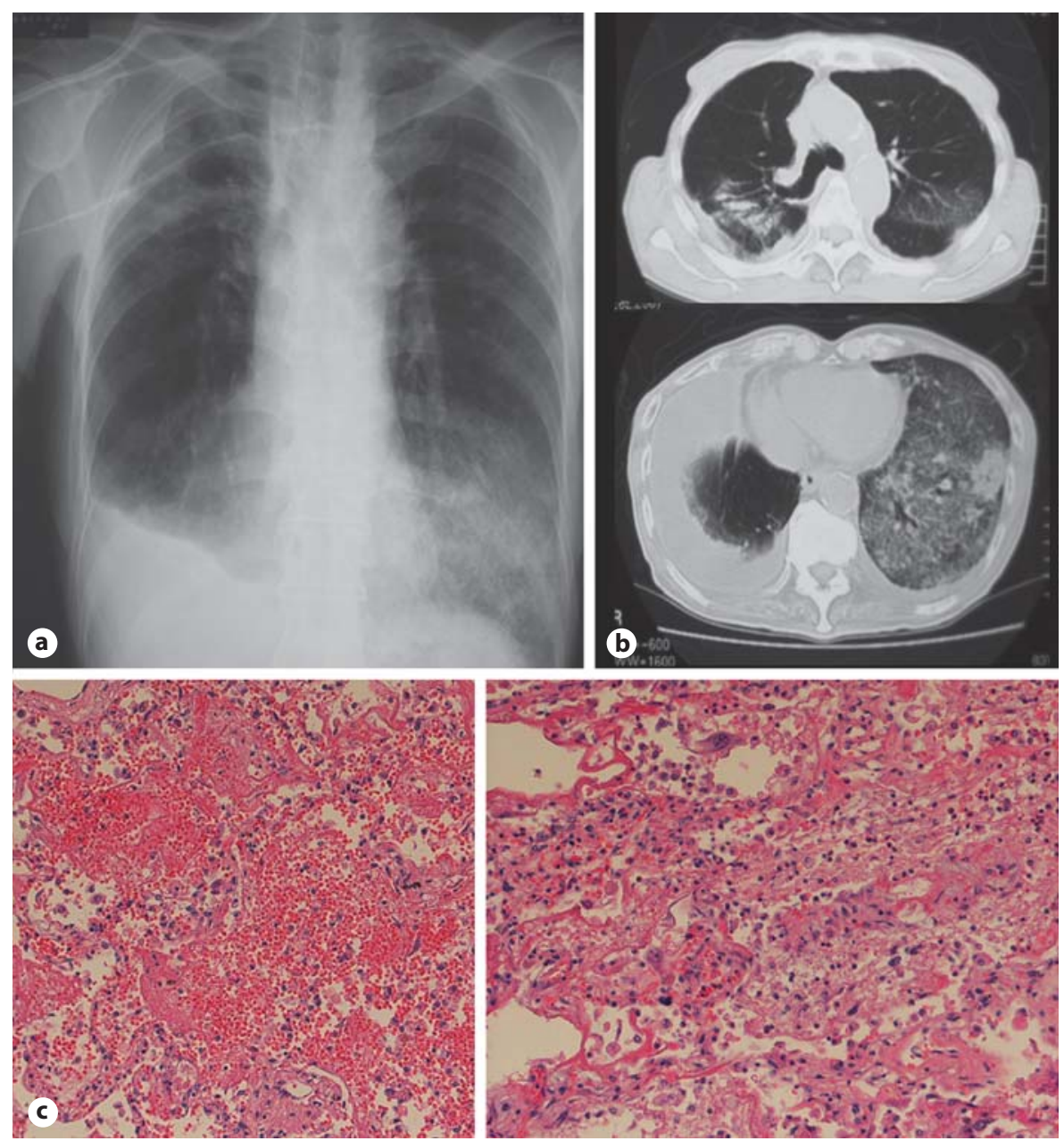

lar disease were excluded by culture findings, bronchoscopy, serologic markers, clinical course and autopsy findings.

Orwell et al. [4] were the first to report mitomycin$\mathrm{C}$-induced lung toxicity, and cases demonstrating this toxicity have been accumulated. The symptoms are progressive dyspnea and a dry cough. The incidence has been estimated to be $5-10 \%$ of patients receiving systemic mitomycin $\mathrm{C}$ administration [5]. The pathological features are characterized as: diffuse alveolar damage [6], acute permeability edema [7] and pulmonary fibrosis [4]. It is widely known that mitomycin C sometimes causes critical adverse effects in the lung when administered systemically. In accordance with previous reports, pathological examination of this case demonstrated diffuse alveolar damage with the hyaline membrane in the lung, which was followed by fatal respiratory failure. However, Verweij et al. [8] reported that mi-
tomycin-C-induced lung toxicity occurred in a dosedependent manner. Indeed, intravesical mitomycin $\mathrm{C}$ chemotherapy is characterized by a lower incidence of adverse systemic effects compared to that of systemic chemotherapy, due to the low rate of systemic mitomycin $\mathrm{C}$ absorption during intravesical administration [2]. However, there are reports describing systemic side effects such as leucopenia [9] and interstitial pneumonia [10] after intravesical mitomycin C chemotherapy. Therefore, we should consider this interstitial pneumonia as a potential systemic side effect of intravesical mitomycin $\mathrm{C}$ chemotherapy despite the low systemic absorption rate of mitomycin $\mathrm{C}$.

Although Neubauer et al. reported intravesical mitomycin-C-induced pulmonary fibrosis in 1987 [10], that case was clinically different from our case. They diagnosed the patient mainly by chest X-ray and pulmonary function tests. According to their description of patho- 
logical findings, pulmonary fibrosis was not demonstrated. Our report provides more information, including the pathological findings. Thus, we consider this to be the first case of pathologically confirmed interstitial pneumonia due to intravesical mitomycin $\mathrm{C}$ chemotherapy.
In conclusion, we have described here the first pathologically confirmed case of interstitial pneumonia (diffuse alveolar damage) caused by intravesical mitomycin $\mathrm{C}$ chemotherapy. Doctors should be aware that intravesical mitomycin $\mathrm{C}$ chemotherapy may cause fatal interstitial pneumonia.

\section{References}

1 Shapiro O, Jones K, Wang C, Landas S, Haas GP: Risk of post-operative intravesical mitomycin C instillation following transurethral bladder tumor resection. Can J Urol 2006;13: 3317-3320.

2 Mostafid AH, Rajkumar RG, Stewart AB, Singh R: Immediate administration of intravesical mitomycin $\mathrm{C}$ after tumour resection for superficial bladder cancer. BJU Int 2006; 97:509-512.

-3 Bolenz C, Cao Y, Arancibia MF, Trojan L, Alken P, Michel MS: Intravesical mitomycin C for superficial transitional cell carcinoma. Expert Rev Anticancer Ther 2006;6:12731282.

4 Orwoll ES, Kiessling PJ, Patterson JR: Interstitial pneumonia from mitomycin. Ann Intern Med 1978;89:352-355.
5 Okuno SH, Frytak S: Mitomycin lung toxicity: acute and chronic phases. Am J Clin Oncol 1997;20:282-284.

6 Buzdar AU, Legha SS, Luna MA, Tashima CK, Hortobagyi GN, Blumenschein GR: Pulmonary toxicity of mitomycin. Cancer 1980;45:236-244.

7 Sheldon R, Slaughter D: A syndrome of microangiopathic hemolytic anemia, renal impairment, and pulmonary edema in chemotherapy-treated patients with adenocarcinoma. Cancer 1986;58:1428-1436.
8 Verweij J, van Zanten T, Souren T, Golding R, Pinedo HM: Prospective study on the dose relationship of mitomycin C-induced interstitial pneumonitis. Cancer 1987;60:756761.

9 Issell BF, Prout GR Jr, Soloway MS, Cummings KB, Brannen G, Veenema R, Flanagan M, Block NL, Summers JL, Levin EA, et al: Mitomycin C intravesical therapy in noninvasive bladder cancer after failure on thiotepa. Cancer 1984;53:1025-1028.

10 Neubauer H, Blech M, Wilhelms E, Criee CP, Neuhaus KL, Truss F: Pulmonary fibrosis following instillation of mitomycin $\mathrm{c}$ in the urinary bladder (in German). Urologe A 1987;26:41-43. 